\title{
DRIVER PERFORMANCE ASSESSMENT WITH A CAR FOLLOWING MODEL
}

\author{
Erwin R. Boer \\ LUEBEC \\ San Diego, California, USA \\ E-mail: erwinboer@san.rr.com \\ Nicholas J. Ward, Michael P. Manser \\ Department of Mechanical Engineering \\ University of Minnesota \\ Minneapolis, Minnesota, USA \\ Tomohiro Yamamura, Nobuyuki Kuge \\ Nissan Motor Co. Ltd. \\ IT \& ITS Engineering Department \\ Yokusuka, Japan
}

\begin{abstract}
Summary: Driver performance is generally quantified by the state of the vehicle relative to the local road and traffic environment. Unfortunately these vehiclestate-based metrics are limited in their diagnostic value when it comes to trying to assess how: (i) drivers individually adopted different control strategies, (ii) how they individually adapted to the issues under investigation (e.g., in-vehicle task execution, driver support system exposure, or impairment), or (iii) why drivers individually were more or less affected by the factor under study. By representing a driver's behavior in an identifiable computational driver model, insight is gained into how drivers may differentially benefit or be impaired by the condition at hand. Such a model also shows how the myriad of possible performance metrics are all "necessarily" correlated. Based on test track car following data, a driver car following model is introduced and identified for each driver and used to show how drivers differ in their car following control strategies. It is demonstrated that the adopted target time headway (THW) strongly influences the associated control strategy (i.e., effort) as well as the safety margin (i.e., the minimum THWs experienced) and that subjects who adopt a longer target THW also exhibit a lower bandwidth control strategy (i.e., less effort).
\end{abstract}

\section{INTRODUCTION}

Computational driver models embody our understanding of how drivers go about performing a particular task. A model provides a framework that logically links the vast number of possible metrics that can be derived from a driver's interactions with a dynamics system. It also offers a means to use a minimal set of measures (e.g., targets or references, control bandwidth, and tolerances or safety margins, model-fit characteristics) that most sensitively and insightfully distinguish across drivers within a condition or within a driver across conditions. Focus here is directed to car following (Boer, 1999), but the same technique is applicable to other driving subtasks such as lane keeping and curve negotiation. It is necessary to recognize that often used metrics such as mean THW or standard deviation in THW can be achieved in a number of ways, thus providing little insight into how drivers approached the task(s) at hand. For example, a 
larger variability in THW can be caused by a more relaxed tolerance range or by a greater struggle in control, which have very different model coefficient changes associated with them, and result in very different interpretations and usages of the results. A car following control model identified for each driver offers a means to extract a deeper understanding of the driver's behavior and thus a better chance at designing a more appropriate training program, selection criterion, or support system. A car following model is introduced and a few of its associated metrics (the full set requires a much larger forthcoming journal paper) are presented and discussed in the context of revealing the individual differences observed in a car following task on a naturalistic test track.

\section{EXPERIMENTAL DESIGN}

A car following driver behavior study was conducted with subjects in two age groups 25-45 and 65-85. Sixteen subjects drove a fully instrumented Infinity Q45 following a partly instrumented Infinity M45 (see picture insert of the test track). The M45 automatically followed a naturalistic speed profile on the straight segments and automatically decelerated for curves (using DGPS and a digitized map of the test track). Upon curve exit, it performed an exaggerated acceleration to a speed higher than the target followed by a deceleration to the speed dictated by the natural speed profile. In this study, we examined the portions of data where the M45 followed the

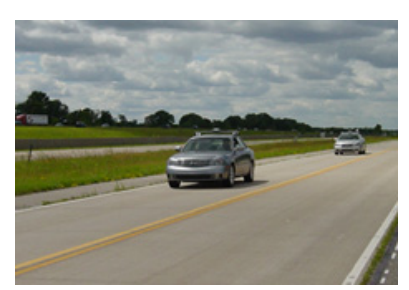
naturalistic speed profile. The naturalistic speed profile was generated based on the power spectral density of vehicle speeds observed on the San Diego freeway. Occasionally (twice per lap), the M45 would engage in a deceleration between 1 and $3 \mathrm{~m} / \mathrm{s}^{2}$ for a period of between 3 and five seconds. Each subject circled the track 4 times over a period of about 20 minutes. The bandwidth of the naturalistic lead vehicle speed profile is sufficient to identify a full driver model; this is not the case for the sinusoidal lead vehicle speeds dictated by the coherence technique of which our approach is a generalization (Brookhuis et al., 1994; Boer et al., 2005).
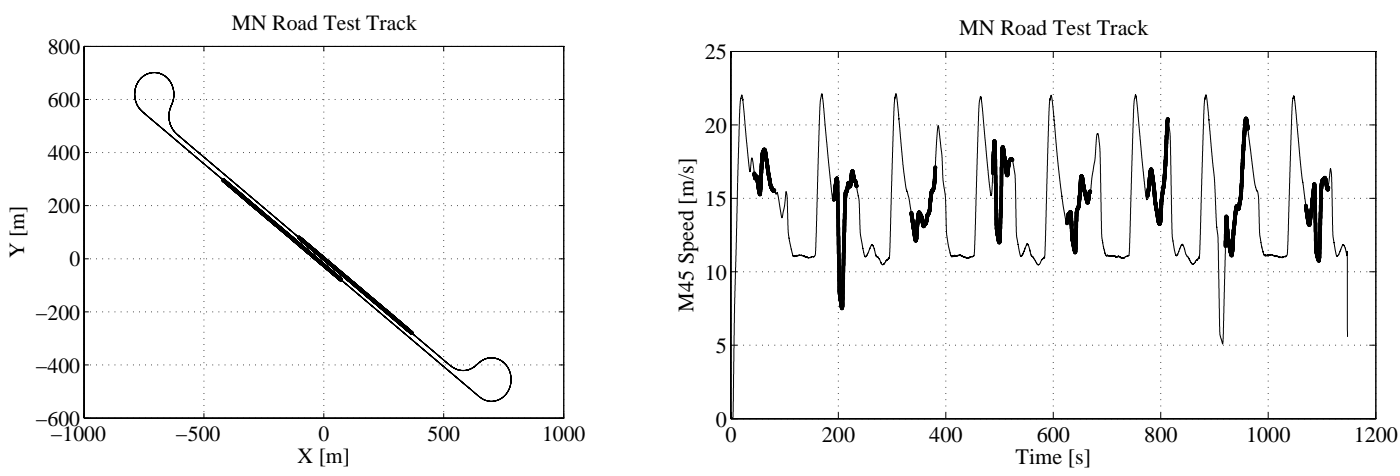

Figure 1. Top view of the test track (left panel). The bold sections in the left panel indicate the straight segments used for the study; they avoid pre-curve deceleration and post-curve acceleration, as can be seen in the corresponding bold section in the speed profile of the lead vehicle (Infinity M45). 


\section{DRIVER VEHICLE MODEL}

The full driver vehicle car following model (Figure 2) requires a vehicle model that accurately captures the relationship between pedal depressions and speed fluctuations. Given that the speeds are mostly above $10 \mathrm{~m} / \mathrm{s}$ (except during a few of the M45 braking events), the assumption of a fixed highest gear is reasonable. The driver as a controller is assumed to consists of three loops that contribute to the final pedal position: (i) a proportional controller on the distance error signal, (ii) a proportional controller on the speed error signals, and (iii) a bias term that produces a pedal depression that would yield the current lead vehicle speed in steady state if maintained sufficiently long. The errors cause a change in pedal position away from steady state.

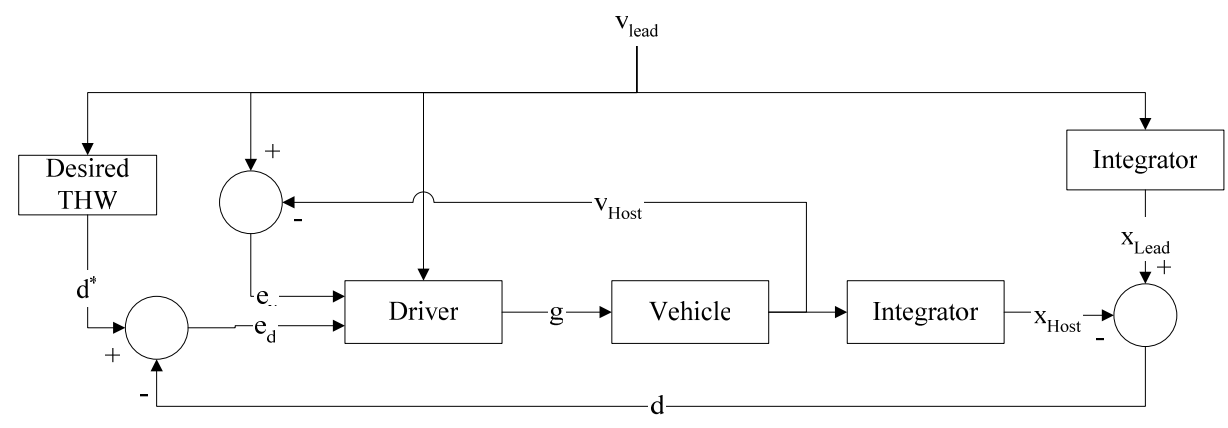

Figure 2. Driver vehicle car following model with lead vehicle speed as input

\section{Vehicle Dynamics Model}

The vehicle dynamics model is constructed around the steady state speed that is reached for a given gas pedal depression (see Figure 3 for the identified relationship) and an acceleration or deceleration rate that depends on how far the gas pedal depression deviates from the one that corresponds to the steady state pedal depression associated with the current speed (i.e., if the current speed were the steady state speed). The vehicle model is described by the following equation when the pedal value $g_{n}$ is positive (a single pedal signal is used; negative when the brake is pressed and positive when the gas is depressed; the delay to go from gas to brake pedal is ignored in this version of the model):

$$
\begin{aligned}
v_{s s}(g) & = \begin{cases}g<\zeta & \alpha_{0}+\alpha_{1} g+\alpha_{2} g^{2} \\
g \geq \zeta & \alpha_{0}+\alpha_{1} \zeta+\alpha_{2} \zeta^{2}\end{cases} \\
v_{n}^{h} & =v_{n-1}^{h}+\lambda\left(v_{s s}\left(g_{n-1}\right)-v_{n-1}^{h}\right)
\end{aligned}
$$

where $\zeta$ is simply the pedal value at the peak of the identified inverted parabola with $\alpha$ coefficients, and

$\lambda= \begin{cases}c_{0}^{+}, & g_{n} \geq 0 \wedge\left(v_{n}^{h, s s}\left(g_{n-1}\right)-v_{n-1}^{h}\right) \geq 0 \\ c_{0}^{-}, & g_{n} \geq 0 \wedge\left(v_{n}^{h, s s}\left(g_{n-1}\right)-v_{n-1}^{h}\right)<0\end{cases}$

Gas pedal depression of about 0.5 (i.e., $\zeta$ ) are not highly representative because of the severely limited number of observations.

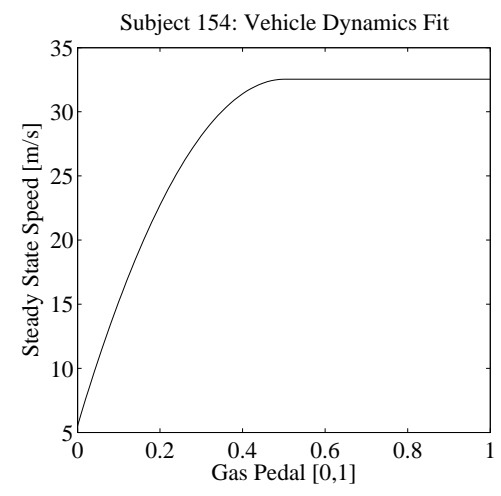

Figure 3. Relationship $v_{s s}(g)$ between gap pedal position and steady state vehicle speed 
If the brake pedal is depressed (i.e., $g_{n}$ has become negative), the following equation is used: $v_{n}^{h}=a_{1} v_{n-1}^{h}+b_{0} g_{n}$, where the vehicle speed decays (i.e., engine, air and road friction) even when the brake is not depressed and it is assumed that the brake signal magnitude is linearly related to deceleration rate. The identified coefficients based on a $200 \mathrm{~ms}$ time-step are: $\alpha_{0}=5.5169$, $\alpha_{1}=107.4189, \alpha_{2}=-106.7421, b_{1}=1.9101, c_{0}^{+}=0.0135, c_{0}^{-}=0.0059, a_{1}=0.996$. This intuitive simple model is sufficient for human vehicle interaction car following research. Further details and specifics on the gradient search method used for identification are given in Boer et al., 2005.

\section{Driver Model}

The driver model shown in Figures 2 and 4 is a straightforward proportional controller on distance deviation from target distance (i.e., $v^{l} \tau^{*}$ where $v^{l}$ is the lead vehicle speed and $\tau^{*}$ the target THW) and a proportional controller on relative velocity. The error-free (i.e., zero velocity at the target THW) gas pedal depression is based on the current lead vehicle speed using the inverse of the relationship shown in Figure 3. The complete set of equations comprising the driver model is:

$$
\begin{aligned}
x_{n}^{h} & =x_{n-1}^{h}+v_{n-1}^{h} T_{s} \\
x_{n}^{v} & =x_{n-1}^{v}+v_{n-1}^{v} T_{s} \\
d_{n} & =x_{n}^{v}-x_{n}^{h} \\
e_{n}^{d} & =\tau^{*} v_{n-1}^{l}-d_{n-1} \\
\Delta g_{n}^{d} & =k_{0} e_{n}^{d} \\
e_{n}^{v} & =v_{n-1}^{l}-v_{n-1}^{h} \\
\Delta g_{n}^{v} & =c_{0} e_{n}^{v} \\
g_{n}^{s s} & =v_{s s}^{-1}\left(v_{n-1}^{l}\right) \\
g_{n} & =g_{n}^{s s}+\Delta g_{n-\delta}^{d}+\Delta g_{n-\delta}^{v}
\end{aligned}
$$

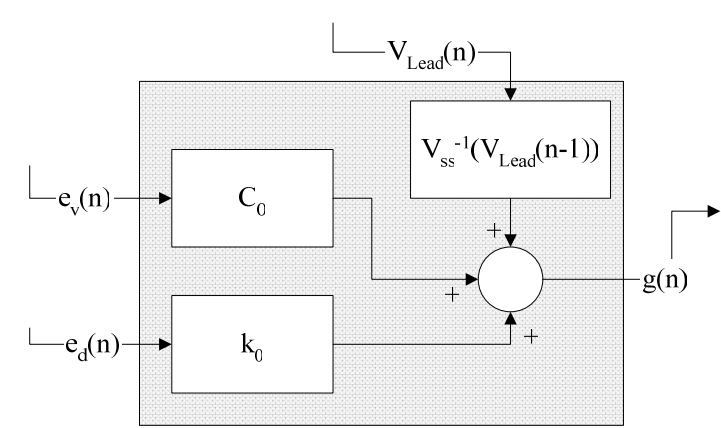

Figure 4. Schematic of the three driver control channels that affect pedal changes.

Typical values of the three free coeffients $\tau^{*}, k_{0}, c_{0}$ can be found in the results figures below (the adopted time step was again $200 \mathrm{~ms}$ ). A delay $\delta$ of zero was used because the very minor improvement in model fit with a non-zero gain did not warrant the extra model coefficient. Finally, it is assumed that the driver has a perfect internal representation of the relationship in Figure 3 and that (s)he uses it to generate a steady state gas pedal position on top of which the pedal is depressed more or less depending on the errors in distance and relative velocity. The driver model coefficients $\tau^{*}, k_{0}, c_{0}$ were identified using a gradient search method (Matlab function "fminsearch") similar to the approach detailed in Boer et al., 2005. The vehicle state was first initialized using observations and the model with estimated coefficients was run through the entire data set (i.e., including curves) to yield a fit value for the current iteration. The adopted fit function for the gradient search was the squared difference between the modelpredicted and observed distance between host and lead vehicle. Figure 5 shows a typical model fit. 

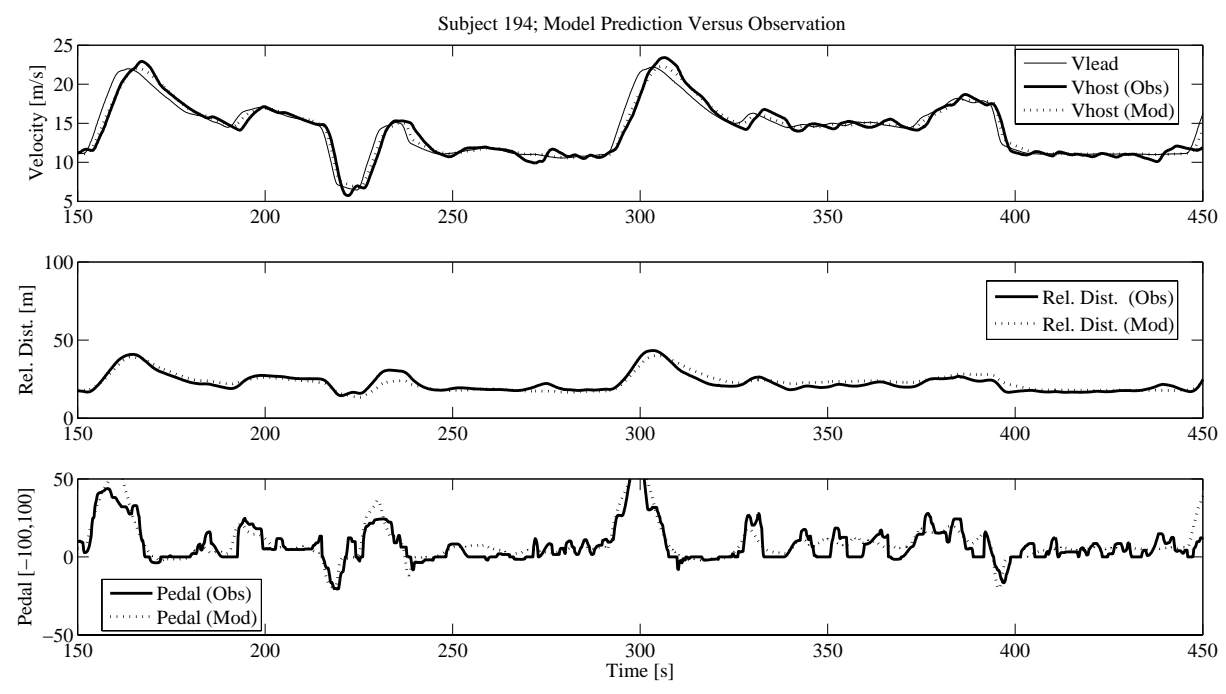

Figure 5. Typical model fit for high bandwidth (see text below for explanation) subject 194.

\section{RESULTS AND DISCUSSION}

The coefficients of the identified models for each subject show a wide range of individual differences in drivers' car following behavior. The main difference is the adopted target THW as shown along the abscissa of the panels in Figure 6. Interestingly, but not entirely unexpectedly, the distance and relative velocity error gains show a strong correlation with the adopted target THW as shown in the right two panels of Figure 6. Drivers who adopt a longer target THW can also accept larger THW deviations from this target, thus enabling them to adopt a lower effort control strategy (i.e., lower control bandwidth as will be discussed below). To anchor the discussions, subjects 154 and 194 with opposite control strategies are selected to exemplify the differences in response characteristics associated with the differences in target THW and error gains. (Note that the only comment on gender effects mentioned in this paper is that it appears that females stay away from short target THWs and thus high bandwidth control strategies.)
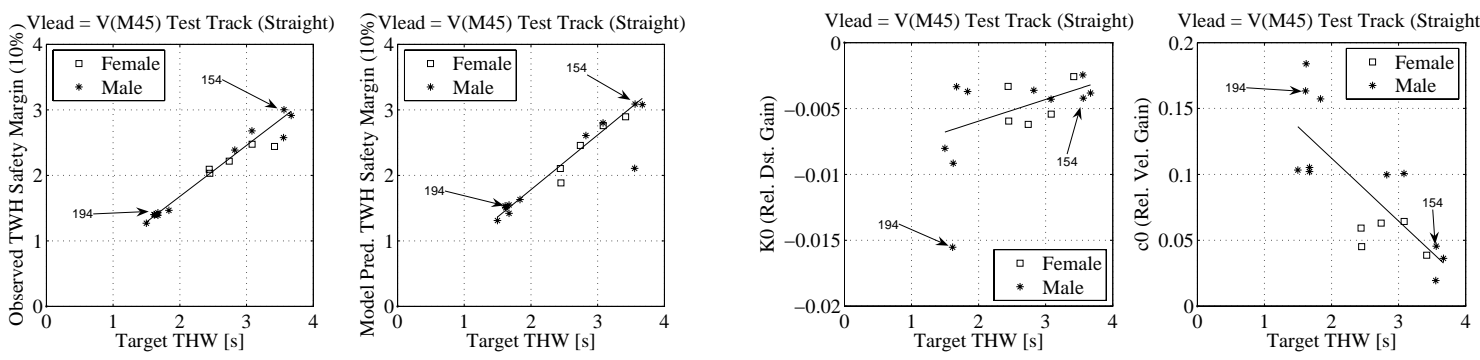

Figure 6. The left two panels show the strong correspondence between observed (Panel 1) and model-generated (Panel 2) lower range of THWs (i.e., 50 percentile - 10 percentile) and the target THW. The right panels show the value of the identified coefficients $k_{0}$ and $c_{0}$ against the target THWs. The reference subjects 154 and 194 are indicated in each panel, as are results for males and females. 
The differences in target THW and error gains are best captured by the bode plots shown in Figure 7. The bode plots shown in the left panel of the figure indicate the transfer function (i.e., dynamic relationship) between the lead vehicle speed and the host vehicle speed for the two anchor subjects. These bode plots show how strongly (gain) and how rapidly (phase) a subject responds to a particular sinusoidal lead vehicle speed fluctuation (Jagacinski \& Flach, 2003). A gain of 1 and a phase of 0 means that the driver is able to immediately and perfectly match the lead vehicle speed with the host vehicle (this is necessarily so for steady state speeds or equivalently frequency zero lead vehicle speed because otherwise the two cars would eventually crash). We see that for higher frequency components in the lead vehicle speed profile, subjects do not try to or cannot follow the lead vehicle speed immediately and perfectly as indicated by a drop in gain and an increase in phase. This means that if the lead vehicle decelerates rapidly (i.e., a high frequency component in the lead vehicle speed profile), that a longer phase lower gain (i.e. lower bandwidth control strategy) will result in a longer delay and a less severe deceleration than what a high bandwidth control strategy would exhibit. The negative consequence of the low bandwidth control strategy is that the gap will decrease more than with the high control bandwidth strategy (this is also reflected in the bode plot of the transfer function between v lead and the gap as shown in the right panel of Figure 7). However, because the low bandwidth control strategy is associated with a longer target THW (as seen in the left most panel of Figure 8), the actually experienced minimum gap or THW is still greater than what high-bandwidthcontrol-strategy subjects experience with their lower target THWs. This is shown in Figure 8's right panel.
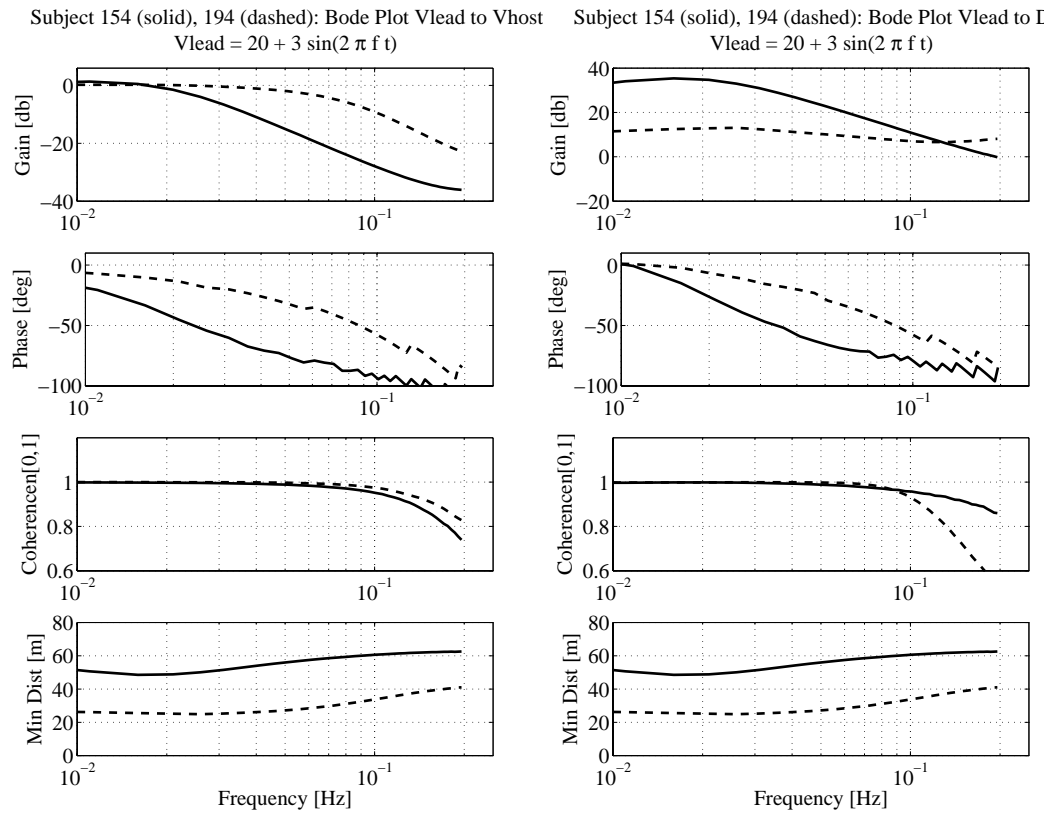

Figure 7. Bode plots for anchor subjects 154 and 194. Left panel shows transfer function from $v$ lead to $v$ host where the right panel shows the corresponding transfer function from $v$ lead to the distance between the two vehicles. The top row shows the gain, the second row the phase, the third the coherence (i.e., how linear is the transfer from a sinusoidal $v$ lead signal to either the host vehicle or the gap distance). The bottom row shows the minimum distance that is reached (see text for details). 
The bode-plots in Figure 7 were created with simulation runs of the identified subject models. The model was run with a lead vehicle speed profile $v^{l}(t)=20+3 \sin (2 \pi f t)$ for a range of frequencies. The gain, phase, and coherence between this input lead vehicle profile and the model generated host vehicle profile were computed and are respectively shown for each frequency $f$ in the top three row of the panels in Figure 7. The bottom row shows the minimum distance between the two vehicles observed during the simulation run at each frequency. The $3^{\text {rd }}$ panel in Figure 8 shows the frequency at which the smallest minimum distance $\left(4^{\text {th }}\right.$ panel $)$ is observed.
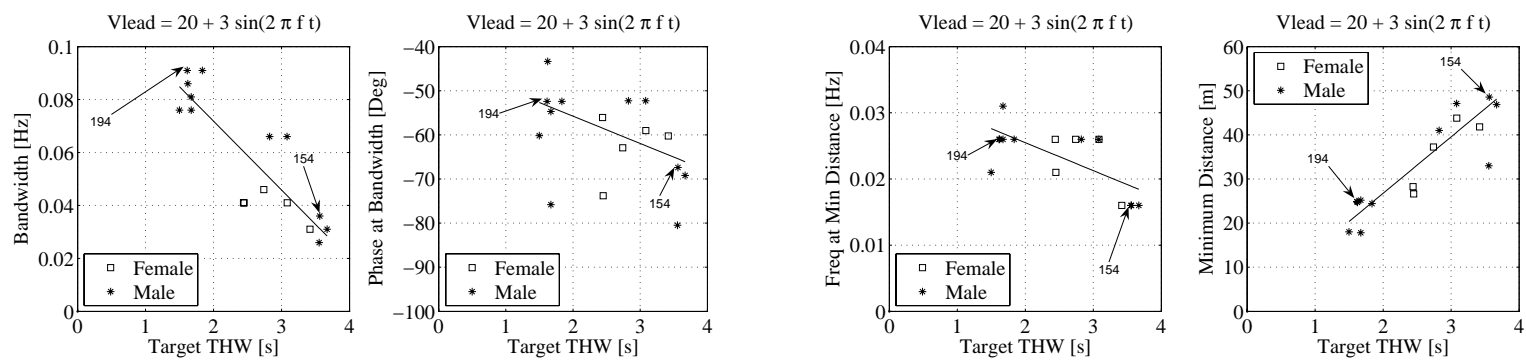

Figure 8. The left two panels show the relationship between bandwidth and phase as a function of target THW. The right two panels show the safety margin as a function of target THW.

\section{CONCLUSIONS}

Drivers who adopt a longer target THW also exhibit a less effortful control strategy as reflected in their lower bandwidth (i.e., less effort allocated to control high frequency changes in lead vehicle speed rapidly and accurately). The observed subjects spanned the full range of intermediate target THWs and control strategies. These results clearly show that a larger variability in observed THW is not necessarily a sign of degraded control performance but that it is a logical consequence of a different control strategy (i.e., the result of a lower effort control strategy rather than a sign of greater struggle). These types of insights are difficult to glean from vehicle state metrics alone.

It is hypothesized that subjects adopt a target THW and settle into a control strategy that offers a sufficient level of THW control given the expected range of lead vehicle decelerations. In this study, as well as in the simulator-based distraction study discussed in Boer et al. (2005), the target THW and the bandwidth of the adopted control strategy are closely correlated. Furthermore in the Boer et al. (2005) study, it appears that subjects cope with the visually demanding in-vehicle tasks by simply shifting their strategy towards longer target THWs and settle into a control bandwidth that ordinarily would be observed at that THW under nondistracted baseline driving conditions. In the Boer et al. (2005) distraction study it is also shown that the model fit deteriorates as subjects engage in a secondary task, indicating that the implicit assumption of continuous control no longer holds as distracted subjects engage in an intermittent control strategy. (The car following model has now been expanded to include a perceptual gating mechanism.) The proposed model-based analysis offers a coherent framework and an insightgenerating language for predicting, assessing, and interpreting driver behavior.

Besides these effects of in-vehicle task performance or other distractions, the large individual differences in control strategies suggest several hypotheses about the impact of support system: 
(i) drivers who adopt a long target THW are expected to be minimally affected by a car following support system that is tuned for much lower THWs, and (ii) drivers who adopt a short target THW may be annoyed by a car following support system that is tuned for a longer THW. This suggests that driver adaptive support may be necessary to achieve universal benefit and acceptance (ignoring the possibility that drivers may simply adopt the target THW "imposed" by the system; a condition that also can easily be recognized with the proposed model-based approach). It is expected that a deeper insight into the control theoretic sources behind observed the individual differences will not only increase our understanding of human functioning but also yield more optimally adapted driver support programs and systems.

\section{ACKNOWLEDGMENTS}

The data used in this work are part of a larger driver support system research study at UMN that was fully funded by Nissan Motor Co., LTD. The authors thank Nissan for its support to develop new techniques that quantify performance from a driver centered perspective.

\section{REFERENCES}

Boer, E.R. (1999). Car following from the driver's perspective. Transportation Research Part F, 2: 201-206.

Boer, E.R., Ward, N.J., Manser, M.P., Kuge, N. (2005). Driver-Model-Based Assessment of Behavioral Adaptation. In: Proceedings of JSAE Spring, May 16-20, Yokohama, Japan.

Brookhuis K.A., de Waard D., Mulder L.J. (1994). Measuring driving performance by carfollowing in traffic. Ergonomics, 37: 427-34.

Jagacinski, R.J., Flach, J.M. (2003). Control Theory for Humans. Mahwah, NJ: Lawrence Erlbaum. 